\title{
CARACTERIZAÇÃO DA FALA DE CRIANÇAS DE 4 A 6 ANOS DE CRECHES PÚBLICAS
}

\section{Speech characterization of 4- to 6-year-old children}

\author{
Lidia Lourenço Cunha Bragança ${ }^{(1)}$, Stela Maris Aguiar Lemos ${ }^{(2)}$, \\ Cláudia Regina Lindgren Alves ${ }^{(3)}$
}

\section{RESUMO}

Objetivo: caracterizar a fala de crianças de 4 a 6 anos de duas creches públicas de Belo Horizonte e classificá-la segundo dois critérios de referência nacional. Método: foram avaliadas 60 crianças em relação à fala. Foram realizadas análises relativas ao inventário fonético, segundo a ordem de ocorrência proposta por dois autores, e à associação entre as classificações. Resultados: a prevalência de inadequações de fala encontrada foi alta nas duas creches, sendo que $45 \%$ e 53,3\% das crianças foram consideradas com fala inadequada segundo as classificações 1 e 2 respectivamente. Houve boa concordância entre as duas classificações (coeficiente de Kappa $=0,768$ ). Não houve associação com significância estatística entre os sexos e as idades. As alterações de fala mais prevalentes foram presença assistemática e substituição, os únicos fonemas com 100\% de ocorrência na amostra total de crianças avaliadas foram /t, k, m, n, y/ , os de menor ocorrência foram, o arquifonema /R/ $(30 \%)$ o fonema / / / (63,3\%) e /// (68,3\%) em encotro consonantal. Conclusão: na faixa etária de 4 a 6 anos as crianças ainda produzem vários fonemas de maneira assistemática, ocorrendo alterações como substituições e omissões. Deve-se levar em conta fatores que podem ter influenciado os resultados, como local da pesquisa, classe econômica e características individuais. Outros estudos sobre a aquisição fonológica são necessários, contribuindo para programas de intervenção precoce e ações preventivas em ambientes escolares.

DESCRITORES: Fala; Creches; Pré-Escolar

\section{INTRODUÇÃO}

A comunicação oral, que pode ser traduzida por boa inteligibilidade de fala, influencia a inserção, ascensão social, aprendizagem e auto imagem dos indivíduos desde o início do seu desenvolvimento. A fala é o principal meio de comunicação entre as pessoas e dificuldades nesta área podem repercutir negativamente no desenvolvimento social nas

(1) Aluna do Curso de Fonoaudiologia da Universidade Federal de Minas Gerais, Belo Horizonte, MG.

(2) Fonoaudióloga; Professora Adjunta da Universidade Federal de Minas Gerais, Belo Horizonte, MG; Doutora em Distúrbios da Comunicação Humana (Fonoaudiologia) pela Universidade Federal de São Paulo.

(3) Médica; Professora adjunta do Departamento de Pediatria da Faculdade de Medicina da Universidade Federal de Minas Gerais, Belo Horizonte, MG; Doutorado em Ciências da Saúde pela Universidade Federal de Minas Gerais.

Conflito de interesses: inexistente diversas fases da vida, caso não sejam precocemente conhecidas e tratadas ${ }^{1,2}$.

A aquisição da fala envolve o conhecimento dos sons e de como esses, são organizados dentro do sistema motor oral. Ela é considerada normal quando o domínio do sistema fonológico ocorre espontaneamente, dentro de uma determinada faixa etária comum a maior parte das crianças. Essa faixa etária se estende na fase pré-escolar, dos 4 aos 6 anos $^{3}$.

Abordando a ordem de aquisição dos fonemas, entre os pesquisadores não há consenso quanto à idade de ocorrência de cada fonema, mas sabe-se que as plosivas, nasais e fricativas são adquiridas primeiro, seguidas pelos arquifonemas /S/ e /R/, por último são adquiridos os encontros consonantais com $/ \mathrm{s} / \mathrm{e} / / /^{4-6}$. A fase de maior expansão do sistema fonológico ocorre entre 1 ano e 6 meses e 4 anos. Nessa fase podem ocorrer omissões e substituições de fonemas, mas é esperado que aos 
5 anos, as crianças já sejam capazes de produzir todos os sons da língua corretamente, apesar de variações individuais serem evidenciadas ${ }^{6}$. Contudo, pesquisas têm demonstrado alta prevalência de alterações de fala em crianças desta faixa etária, em que aproximadamente $10 \%$ das crianças em idade pré-escolar apresentam desvios fonológicos ${ }^{7-9}$.

A linguagem oral é o repertório básico para o processo de alfabetização e por isso o desenvolvimento da fala está ligado à linguagem escrita. Verificou-se que crianças que apresentam atraso de aquisição fonológica na primeira infância, têm certa tendência à continuidade de dificuldades no desenvolvimento da escrita e consequentemente, no rendimento escolar ${ }^{8,9}$. Dificuldades na aquisição da linguagem nos padrões normais são, quase sempre, indícios de problemas de desenvolvimento subjacente $^{10}$.

Pesquisas evidenciam grande proporção de crianças no ensino fundamental com alterações de fala ${ }^{11,12}$. Um estudo com 523 escolares de $1^{\text {a }}$ a $4^{\underline{a}}$ série de uma escola estadual de São Paulo, encontrou prevalência de $37,1 \%$ de alterações de fala, verificou-se também que a percepção do professor em relação a essas alterações é baixa ${ }^{13}$. Isso demonstra a necessidade de ações preventivas em crianças ainda na fase pré-escolar. É fundamental que os educadores tenham conhecimento da prevalência de alterações de fala e sejam capacitados a lidar com questões relacionadas ao desenvolvimento de fala, para que, programas de estimulação da linguagem oral sejam realizados e as crianças identificadas com alterações de fala sejam encaminhadas ao tratamento o mais precocemente possível, evitando assim, futuras dificuldades de aprendizagem ${ }^{8,13}$.

Estudar o desenvolvimento da fala de crianças frequentadoras de creches é importante, pois auxilia a identificação precoce dessas alterações e na criação de medidas de prevenção nestes ambientes. Pensando nisso, o objetivo do presente estudo foi caracterizar a fala de crianças de 4 a 6 anos de duas creches públicas de Belo Horizonte e classificá-la segundo dois critérios de referência nacional, discutindo a ocorrência dos fonemas e a concordância entre as classificações.

\section{MÉTODO}

Trata-se de estudo transversal comparativo com amostra de conveniência, em que foram realizadas avaliações de fala em crianças da faixa etária de 4 a 6 anos frequentadoras de duas creches. A creche está localizada na Regional Leste, e a creche B na Regional Nordeste. O recrutamento para a participação na pesquisa foi feito por meio de carta, que os pais das crianças receberam como convite para participarem da pesquisa. Ao serem abordados no horário de entrada ou saída do filho na creche, foram informados quanto ao caráter voluntário do estudo, seus objetivos e repercussões.

Foram incluídas no estudo 60 crianças de ambos os sexos, na faixa etária de 4 anos a 5 anos e 12 meses, regularmente matriculadas nas creches, no $1^{\circ}$ ou $2^{\circ}$ período primário, cujos responsáveis assinaram o Termo de Consentimento Livre e Esclarecido (TCLE). A coleta de dados foi realizada no período de agosto a dezembro de 2009.

O desenvolvimento de fala das crianças foi avaliado em uma sala na própria creche em sessão individual de aproximadamente 30 minutos e o instrumento utilizado foi um Álbum fonêmico. Nesta prova foram mostradas figuras à criança solicitando nomeação. As figuras do álbum foram selecionadas pelo critério de posição dos fonemas na palavra. $O$ momento da avaliação foi gravado em áudio para que a análise fosse realizada. A identificação das alterações da fala foi realizada baseada na análise perceptivo-auditiva da examinadora. As omissões, distorções e substituições apresentadas foram listadas em um quadro fonêmico do protocolo.

Para análise, foi realizada quantificação dos resultados em planilha. Considerou-se quanto a emissão: presença sistemática, se o fonema ocorreu com produção correta em todas as emissões; presença assistemática, se em alguma emissão o fonema foi omitido ou produzido incorretamente; substituição/distorção assistemática, se o fonema foi substituído por outro ou foi distorcido em uma ou mais emissões, mas não em todas; substituição/distorção sistemática, se o fonema foi substituído por outro ou foi distorcido em todas as emissões; omissão, se não houve ocorrência do fonema em nenhuma emissão. As variações linguísticas por padrões socioculturais (ex: Fósfu, frósfi em vez de fósforo, brusa em vez de blusa ) não foram consideradas como erro.

Após a análise dos resultados encontrados, a fala foi classificada como adequada ou inadequada segundo a ordem de ocorrência proposta por dois autores. A classificação 1 seguiu a ordem de aquisição fonológica proposta em $1994^{4}$ e a classificação 2 a ordem proposta também em $1994{ }^{5}$ (Figura 1). Essas classificações foram escolhidas por descreverem a ordem de ocorrência de todos os fonemas e serem utilizadas no campo da fonoaudiologia. 


\begin{tabular}{|c|c|c|c|}
\hline \multicolumn{2}{|c|}{ Classificação 1: Wertzner, 2000} & \multicolumn{2}{|c|}{ Classificação 2: Mourão et. al, 1994} \\
\hline 3:0 & $\begin{array}{c}/ p, b, t, d, k, g, f, v, s, z, \int, 3 \\
I, r, m, n, y /\end{array}$ & $2: 6$ a $3: 6$ & $\begin{array}{c}/ \mathrm{m}, \mathrm{n}, \mathrm{p}, \mathrm{b}, \mathrm{t}, \mathrm{d}, \mathrm{k}, \mathrm{f}, \mathrm{v}, \mathrm{l}, \mathrm{s}, \mathrm{z}, \mathrm{3} \\
\mathrm{g}, \mathrm{y}, \kappa, \mathrm{s} / \text {, arquifonema/S/ }\end{array}$ \\
\hline 4:0 & $/ K /$, arquifonema $/ \mathrm{S} /$ & $3: 6$ a $4: 6$ & $/ \mathrm{r} /, \mathrm{cc}(\mathrm{I}) \mathrm{v}$, arquifonema/R/ \\
\hline $5: 6$ & $\{\mathrm{R}\}$ & \multirow{3}{*}{$4: 6$ a $5: 6$} & \multirow{3}{*}{$/ \varsigma /$ em encontro consonantal } \\
\hline $4: 0$ a $5: 0$ & /r/ em encontro consonantal & & \\
\hline $4: 0$ a $6: 6$ & /I/ em encontro consonantal & & \\
\hline
\end{tabular}

Figura 1 - Ordem de ocorrência dos sons, em relação á idade segundo as classificações 1 e 2 da fala

Esse estudo foi aprovado pelo Comitê de Ética em Pesquisa da UFMG, pelo parecer ETIC 202/08.

$\mathrm{Na}$ análise estatística dos dados, foram consideradas variáveis dependentes as classificações $1 \mathrm{e}$ 2 da fala e variáveis independentes, a idade (4 e 5 anos), o sexo (feminino e masculino) e a creche (A e B). Foram obtidos os números absolutos e relativos e estes foram submetidos à análise estatística por meio do Teste Qui-quadrado de Pearson, uma vez que todas as variáveis são categóricas. Para verificar a associação entre as duas classificações de Fala, utilizou-se o teste de McNemar e coeficiente Kappa, para verificar a concordância entre elas. O valor de Kappa foi classificado segundo referenciado na literatura ${ }^{14}$, sendo que o valor de Kappa 0,80 a 1,00 indica concordância excelente; de 0,61 a 0,80, concordância boa; de 0,41 a 0,60, concordância moderada; de 0,21 a 0,40,concordância razoável e de $-1,00$ a 0,20, concordância pobre. A análise dos dados foi realizada no programa Minitab versão 14 e foi adotado nível de significância de $\mathrm{p}<0,05$.

\section{RESULTADOS}

A amostra foi composta por 60 crianças com idade entre 4 anos e 5 anos e 12 meses, sendo que a maior proporção era de crianças do sexo masculino, com idade igual a 4 anos pertencentes a creche A. As características das crianças avaliadas são apresentadas na Tabela 1. Verifica-se, em relação a avaliação de fala, que $45 \%$ e $53,3 \%$ foram consideradas com fala inadequada segundo as classificações 1 e 2 respectivamente.

A Figura 2 mostra a Distribuição de frequências, segundo as classificações 1 e 2 de fala, na amostra geral.

Tabela 1 - Distribuição de frequência da amostra segundo as variáveis

\begin{tabular}{lcc}
\hline Característica & $\mathbf{n}$ & $\%$ \\
\hline Idade $(4,0-6,0)$ & 31 & 51,7 \\
4 anos & 29 & 48,3 \\
5 anos & & \\
\hline Sexo & 28 & 46,7 \\
Feminino & 32 & 53,3 \\
Masculino & & \\
\hline Creche & 46 & 76,7 \\
A & 14 & 23,3 \\
B & & \\
\hline Classificação de fala 1 & 33 & 55,0 \\
Adequada & 27 & 45,0 \\
Inadequada & & \\
\hline Classificação de fala 2 & 28 & 46,7 \\
Adequada & 32 & 53,3 \\
Inadequada &
\end{tabular}

idade, sexo, creche, raf e classificação 1 e 2 de fala

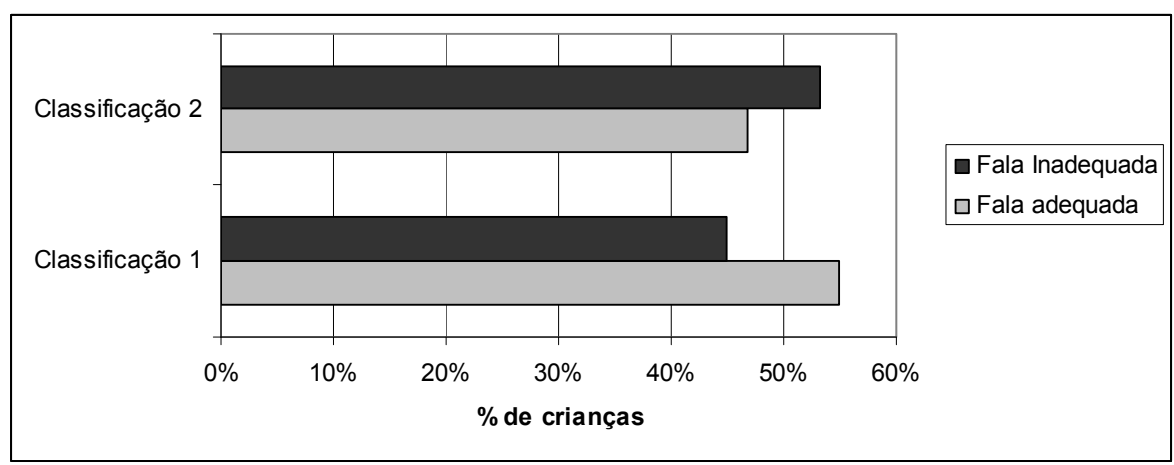

Figura 2 - Distribuição de frequências, segundo as classificações 1 e 2 de fala, na amostra geral 
Na Tabela 2 são apresentadas as características relacionadas à Fala estratificando-se por creche, sexo e idade. Verifica-se que não houve associação com significância estatística entre as classificações (Adequado/Inadequado) e nenhum dos grupos comparados (feminino e masculino, creche A e B, 4 e 5 anos). Observa-se que houve alta ocorrência de inadequações de fala nas duas creches avaliadas.

Na Figura 3 está representada a ocorrência de todos os fonemas na amostra total. Observa-se que os fonemas que mais estiveram presentes assistematicamente foram o arquifonema /R/ e o fonema $/ \mathrm{s} / \mathrm{em}$ encontro consonantal. Os fonemas //3/, /r/ e /z/, foram substituídos com mais freqüência.

Tabela 2 - Distribuição de freqüência - fala estratificada por idade, sexo e creche

\begin{tabular}{|c|c|c|c|c|c|c|c|c|c|c|}
\hline \multirow{3}{*}{ Característica } & \multicolumn{5}{|c|}{ Classificação 1} & \multicolumn{5}{|c|}{ Classificação 2} \\
\hline & \multicolumn{2}{|c|}{ Adequada } & \multicolumn{2}{|c|}{ Inadequada } & \multirow{2}{*}{ p } & \multicolumn{2}{|c|}{ Adequada } & \multicolumn{2}{|c|}{ Inadequada } & \multirow{2}{*}{$\mathbf{p}$} \\
\hline & $\mathbf{n}$ & $\%$ & $\mathbf{n}$ & $\%$ & & $\mathbf{n}$ & $\%$ & $\mathbf{n}$ & $\%$ & \\
\hline \multicolumn{11}{|l|}{ Idade } \\
\hline 4 anos & 16 & 51,6 & 15 & 48,4 & 0,585 & 15 & 48,4 & 16 & 51,6 & 0,782 \\
\hline 5 anos & 17 & 58,6 & 12 & 41,4 & & 13 & 44,8 & 16 & 55,2 & \\
\hline \multicolumn{11}{|l|}{ Sexo } \\
\hline Feminino & 18 & 64,3 & 10 & 35,7 & 0,175 & 15 & 53,6 & 13 & 46,4 & 0,316 \\
\hline Masculino & 15 & 46,9 & 17 & 53,1 & & 13 & 40,6 & 19 & 59,4 & \\
\hline \multicolumn{11}{|l|}{ Creche } \\
\hline A & 24 & 52,2 & 22 & 47,8 & 0,422 & 21 & 45,7 & 25 & 54,3 & 0,775 \\
\hline B & 9 & 64,3 & 5 & 35,7 & & 7 & 50,0 & 7 & 50,0 & \\
\hline
\end{tabular}

Teste Qui-Quadrado de Pearson.
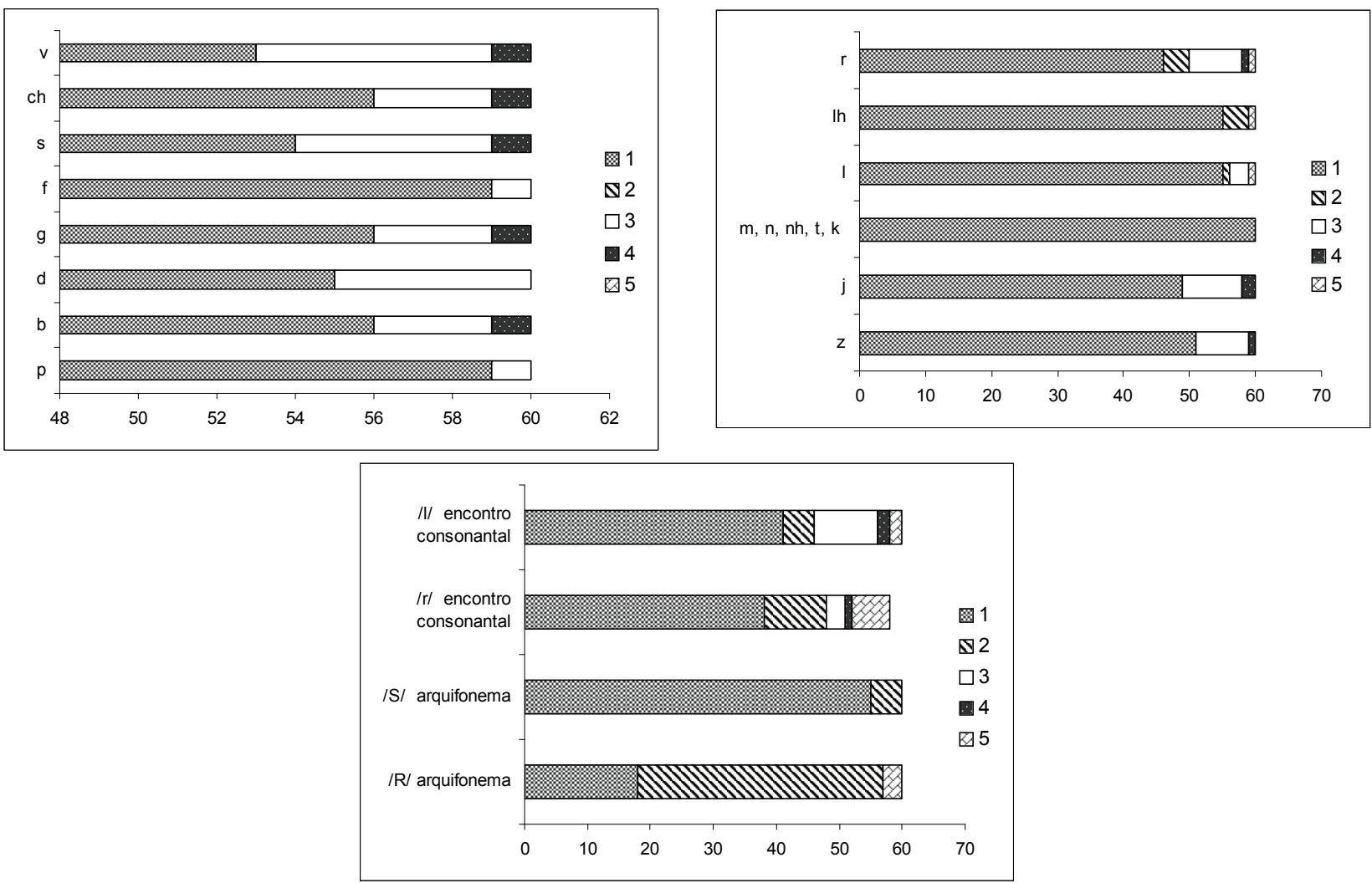

1: Presença sistemática, 2: presença assistemática, 3: substituição/distorção assistemática, 4: substituição/distorção sistemática, 5: omissão

Figura 3 - Gráfico de ocorrência dos fonemas na amostra total 
Tabela 3 - Associação entre as classificações 1 e 2 de fala

\begin{tabular}{ccccccc}
\hline & \multicolumn{5}{c}{ Classificação 2 } \\
\cline { 2 - 5 } Classificação 1 & \multicolumn{2}{c}{ Adequado } & \multicolumn{2}{c}{ Inadequado } & \multirow{2}{*}{ Kappa } \\
\cline { 2 - 5 } & $\mathbf{n}$ & $\%$ & $\mathbf{n}$ & $\%$ & \multirow{2}{*}{0,768} \\
Normal & 27 & 45,0 & 6 & 10,0 & \\
Alterado & 1 & 1,7 & 26 & 43,3 & \\
\hline
\end{tabular}

Teste para dados pareados - Teste de McNemar. (valor-p<0,001)

Verifica-se na Tabela 3 que houve concordância entre as classificações 1 e 2 , sendo que o valor do coeficiente Kappa encontrado indica concordância boa entre as classificações.

\section{DISCUSSÃO}

$\mathrm{Na}$ amostra, verificou-se maior proporção de crianças pertencentes à creche $\mathrm{A}$, devido ao maior número de crianças matriculadas nesta creche. Nota-se, também em relação à amostra total, maior número de crianças do sexo masculino e com idade igual a 4 anos, como a diferença é pequena $(6,6 \%$ para sexo e $3,4 \%$ para idade), isso não desequilibrou a amostra.

Foi encontrada prevalência semelhante de atrasos de fala (57\%) em crianças de 5 anos, em um estudo realizado em Canoas-RS ${ }^{1}$. A proporção de crianças com fala inadequada foi alta nas duas creches, o que demonstra grande incidência de inadequações de fala nas crianças desta faixa etária. Esse achado corrobora com alguns autores que estudaram o desenvolvimento fonológico de pré-escolares em Belo Horizonte e Santa Maria-RS e também encontraram alta prevalência de desvios de fala ${ }^{7,8}$. Outro estudo com crianças de 4 a 6 anos de escolas públicas de Salvador-BA verificou menor prevalência de alterações de fala $(9,7 \%)$ que a encontrada no presente estudo ${ }^{9}$.

Verificou-se que uma porcentagem maior de crianças foi considerada com fala inadequada segundo a classificação 2. Essa diferença pode ter ocorrido, pois de acordo com essa classificação, alguns fonemas, como $/ \mathrm{K} /$, arquifonema $/ \mathrm{S} / \mathrm{e}$ o fonema $/ \mathrm{r} /$ em encontro consonantal, são adquiridos em idade anterior à indicada pela classifica-ção 1 . A classificação 2 dá como referência 5 anos e 6 meses para que o inventário fonético da crianças esteja completo, sendo que a classificação 1 indica o limite até 6 anos e 6 meses ${ }^{4,5}$.

Não há concordância na literatura encontrada, quanto ao sexo em que os desvios de fala mais aparecem. Alguns autores encontraram maior prevalência no sexo masculino ${ }^{7,12,15}$, e outros estudos com pré-escolares não relataram diferenças significativas entre os sexos ${ }^{1,8,16}$. Em Belo Horizonte, em estudo com 297 escolares, também não foi observada diferença em relação ao sexo². Em relação à faixa etária, um estudo sobre a aquisição fonológica de crianças de 2 a 6 anos realizado em São Paulo relatou que poucas mudanças foram observadas entre as idades de 4 a 6 anos $^{8}$, mas outro autor que avaliou a fala de crianças de 4 e 5 anos encontrou maior prevalência de alterações de fala nas crianças de 4 anos $^{16}$. No presente estudo não foi observada diferença estatisticamente significativa na ocorrência de inadequações de fala, em relação ao sexo ou idade.

Quanto a ordem de ocorrência dos fonemas, verificou-se que os únicos fonemas com $100 \%$ de ocorrência na amostra total de crianças avaliadas foram /t, k, m, n, y/,o que não corrobora com alguns estudos que verificaram que praticamente todas as plosivas e nasais são adquiridas antes dos 3 anos e 6 meses de idade ${ }^{4,6}$. Os de menor ocorrência foram, o arquifonema /R/ (30\%) o fonema/ / / (63,3\%) e /// $(68,3 \%)$ em encontro consonantal. Os fonemas $/ \mathrm{p}, \mathrm{b}, \mathrm{d}, \mathrm{g}, \mathrm{f}, \mathrm{s}, \mathrm{S}, \mathrm{v}, \mathrm{z}, \mathrm{3}, \mathrm{l}, \mathrm{K}, \mathrm{f} /$, estavam presentes sistematicamente na maioria das crianças, mas houve grande variabilidade de produção. Outros estudos que investigaram o processo de aquisição fonológica em crianças de 3 a 8 anos do Rio de Janeiro relataram que estes fonemas já estão adquiridos e estabilizados aos 3 anos de idade mas também encontraram variabilidade de produção em grande parte deles ${ }^{6}$.

Observa-se que as alterações de fala mais prevalentes foram presença assistemática e substituição, outros estudos também encontraram essas alterações em crianças de 4 ou 5 anos de idade ${ }^{1,8,16}$. O número de omissões foi pequeno, a literatura encontrada mostra que esse tipo de alteração é menos frequente que alterações como substituição ${ }^{17}$. O fonema mais omitido pelas crianças avaliadas foi o $/ \mathrm{r} /(10 \%)$ em encontro consonantal, grande parte dos estudos sobre o processo de aquisição fonológica encontrados na literatura demonstram que este fonema é um dos últimos a ser adquirido no sistema fonêmico ${ }^{4-7,9,12}$, pois é o som que apresenta maior dificuldade de produção ${ }^{18}$. 
Ao analisar a concordância entre as classificações da fala utilizadas no presente estudo, observase que o valor do coeficiente Kappa $(0,768)$ encontrado indica concordância boa entre elas, isso mostra que as duas classificações indicam ordem de ocorrência semelhante na maioria dos fonemas da língua portuguesa. $\mathrm{Na}$ literatura não há um consenso quanto a ordem de aquisição fonológica, podendo haver variações de acordo com as características individuais das crianças avaliadas ${ }^{3}$, e fatores como região e a classe econômica ${ }^{6,8}$. Ao observar as diferenças na ordem de ocorrência dos fonemas das duas classificações utilizadas no presente estudo, deve-se levar em conta fatores como o ano e local em que foi realizada a pesquisa e também as características das crianças avaliadas, esses fatores podem ter influenciado as diferenças encontradas nos resultados das classificações 1 e 2 .

A grande ocorrência inadequações de fala no presente estudo é um fato preocupante, pois essas dificuldades, se continuadas, podem interferir na comunicação e em outras áreas do desenvolvimento. São necessários outros estudos com amostras maiores, que confirmem os critérios de aquisição fonológica e identifiquem as alterações típicas e atípicas do desenvolvimento para cada fonema do português, levando em consideração fatores significantes. Estudos sobre o desenvolvimento de fala em pré-escolares contribuirão para realização de diagnósticos mais precisos, programas de intervenção precoce e desenvolvimento de ações preventivas em ambientes escolares.

\section{CONCLUSÃO}

O presente estudo mostrou grande ocorrência de inadequações de fala nas duas creches avaliadas e não houve associação com significância estatística entre a ocorrência de inadequações de fala, o sexo e a idade. Também permitiu verificar que na faixa etária de 4 a 6 anos as crianças ainda produzem vários fonemas de maneira assistemática e ocorrem alterações, como substituições e omissões, sendo que o fonema mais omitido pelas crianças avaliadas foi o cc/r/ . A concordância entre as classificações da fala utilizadas no presente estudo foi considerada boa de acordo com o coeficiente Kappa, mostrando que as duas classificações indicam ordem de ocorrência semelhante na maioria dos fonemas da língua portuguesa.

\begin{abstract}
Purpose: to characterize the speech of 4- to 6-year-old children in two public day care centers in Belo Horizonte and classify it according to two national benchmark references. Method: 60 children were evaluated regarding their speech. Analyses were made up on top of the phonetic inventory, according to the order of occurrence proposed by two authors, and the association among the classifications was studied. Results: the rate found for speech mismatch was high in both centers, with $45 \%$ and $53.3 \%$ of the children showing inappropriate speech abilities according to the ratings 1 and 2 , respectively. A good agreement between the two classifications was found (Kappa coefficient $=0.768$ ). No statistically significant association between gender and age was found. The most noted speech disorders were unsystematic presence and replacement, the only phonemes with $100 \%$ of occurrence in the total sample of assessed children were /t, k, m, n, y/. The less frequent phonemes were /R/ $(30 \%), \mathrm{cc} / \mathrm{r} /$ $(63.3 \%)$ and cc/l/ (68.3\%). Conclusion: 4- to 6-year-old children still produce many phonemes in an unsystematic way, occurring speech changes such as replacement and omissions. Factors that might have influenced the results, such as, the research site, economic class and individual characteristics, that should be taken into account. Other studies of phonological acquisition are needed, contributing to early intervention programs and preventive actions in the school environments.
\end{abstract}

KEYWORDS: Speech; Child Day Care Centers; Child, Preschool 


\section{REFERÊNCIAS}

1. ChiariBM, GoulartBNG. Prevalência de desordens de fala em escolares e fatores associados. Rev Saúde Pública 2007;41(5):726-31.

2. Rabelo ATV. Prevalência de alterações fonaudiológicas em crianças de $1^{\circ}$ a $4^{\circ}$ serie de escolas públicas da área de abrangência de um centro de saúde de Belo Horizonte. [Dissertação de mestrado]. Belo Horizonte: Programa de Mestrado Saúde da criança e do adolescente da Universidade Federal de Minas Gerais, 2010.

3. Lamprecht RR. Antes de mais nada In: Lamprecht RR et al.Aquisição fonológica do português: perfil de desenvolvimento e subsídios para terapia. Porto Alegre: Artmed; 2004. p. 17 a 32.

4. Wertzner HF, Aquisição da Articulação: Um estudo em crianças dos três aos 7 anos. Rev Estud Psicol. 1994; 11(1/2):11-21.

5. Mourão LF, Parlato EM, Silvério KCA, Altmann EBC, Chiari BM. Descrição da ocorência dos fonemas da língua portuguesa em pré-escolares. Pró-Fono Revista de Atualização Científica. 1994; 6(1):27-32.

6. Ferrante C, Van Borsel J, Medeiros M. Aquisição fonológica de crianças de classe sócio econômica alta. Rev CEFAC. 2008; 10(4):452-60.

7. Casarim MT. Estudo dos desvios de fala em pré-escolares de escolas públicas estaduais de Santa Maria - RS. [Dissertação de Mestrado]. Santa Maria: Programa de pós-graduação em Distúrbios da Comunicação. 2006.

8. Vitor RM, Martins CC, Desenvolvimento fonológico de crianças pré-escolares da Região Noroeste de Belo Horizonte. Psicologia em Revista. 2007;13(2): 383-98.

9. Cavalheiro LG, Keske-Soares M. Prevalência do desvio fonológico em crianças de 4 a 6 anos de idade [resumo 4]. Pró-Fono Revista de Atualização Científica [Internet]. 2008 [citado 2008 out 13];20(Supl):11-3. [Apresentado no II
Congresso Brasileiro de Fonoaudiologia e Genética dos Distúrbios da Comunicação; 2008; Fortaleza, Ceará]. Disponível em: www.revistaprofono.com.br. 10. Pedroso FS, Rotta NT, Danesi MC, Avila LN, Sávio CB. Evolução das manifestações pré-linguísticas em crianças normais no primeiro ano de vida. Rev Soc Bras Fonoaudiol. 2009;14(1):22-5. 11. Santos LK, Ávila CRB, Cechella C, Morais ZR. Ocorrência de alterações de fala, do sistema sensoriomotor oral e de hábitos orais em crianças pré-escolares e escolares da $1^{\underline{a}}$ série do $1^{\circ}$ grau. Pró Fono Revista de Atualização Científica. 2000; 12 (2), 93-101.

12. Patah LK, Takiuchi N. Prevalência das alterações fonológicas e uso de processos fonológicos em escolares aos 7 anos. Rev CEFAC. 2008; 10(2):158-67

13. Silva MR, Canêdo LB, Marchesan IQ. Alterações de fala em escolares do ensino fundamental: ocorrência, identificação e condutas adotadas. Anais do 16을 Congresso Brasileiro de fonoaudiologia. Set 2008. Campos do Jordão (SP). Disponível em http:// www.sbfa.org.br/portal/anais2008/pg.php\# .

14. Altman DG. Pratical statistics for medical research. London: Chapman and Hall; 1991.

15. Casarin MT, Gindri G, Keske-Soares M, Mota HB. Alterações do sistema estomatognático em distúrbios da fala. Rev. Soc. Bras. Fonoaudiol. 2006; 11(4): 223-30.

16. Farias SR, Ávila CRB, Vieira MM. Relação entre fala, tônus e praxia não-verbal do sistema estomatognático em pré-escolares. Pró-Fono. 2006; 18(3):267-76.

17. Soares K, Blanco APF, Mota, HB. O desvio fonológico caracterizado por índices de substituição e omissão. Rev Brás. Fonoaudiol. 2004; 9:10-8.

18. Wertzner HF, Pagan-Neves LO, CASTRO MM. Análise acústica e índice de estimulabilidade nos sons líquidos do português brasileiro. Rev. CEFAC. 2007; 9(3): 339-50.

http://dx.doi.org/10.1590/S1516-18462011005000053

RECEBIDO EM: 17/08/2010

ACEITO EM: 25/11/2010

Endereço para correspondência:

Stela Maris Aquiar Lemos

Faculdade de Medicina da Universidade

Federal de Minas Gerais - Departamento de

Fonoaudiologia

Av. Prof. Alfredo Balena, 190

Belo Horizonte - MG - Brasil

CEP: 30130-100

E-mail: lidiacunha@msn.com 